\title{
Contenido de grasas, sodio, azúcares y edulcorantes declarados en etiquetado nutricional de golosinas, snacks salados y en bebidas dulces no alcohólicas seleccionadas
}

\author{
Stated content of fats, sodium, sugars and sweeteners in \\ nutritional labeling of sweets, salty snacks and in selected \\ non-alcoholic sweet drinks
}

\author{
Romy Beatriz Hermosilla Soria', Susana F. Sánchez Bernal', Rodrigo Burgos'
}

\section{RESUMEN}

Introducción: La obesidad en niños se asocia también a ingesta de alimentos ricos en grasas, azúcares y sodio, por ello es importante conocer sus niveles en golosinas y las bebidas dulces. Objetivos: Evaluar contenido de nutrientes críticos acorde al Perfil de Nutrientes de OPS en golosinas dulces, snacks salados y bebidas dulces no alcohólicas envasadas, según información del etiquetado nutricional. Materiales y Métodos: Estudio transversal, descriptivo, exploratorios. Se evaluó contenido por porción de grasas totales(GT), saturadas(GS) y trans(GTr), sodio, azúcares libres(AL) y edulcorantes artificiales(EA) en golosinas dulces, saladas, y bebidas dulces no alcohólicas (BDNA) según etiquetado nutricional. Niveles excesivos fueron considerados según "Perfil de nutrientes de OPS". Muestra fue por conveniencia obtenida en 2 bocas de expendio. Se excluyeron postres envasados y golosinas con información poco visible. Resultados: Fueron evaluados 104 alimentos agrupados en: confitería (alfajor, magdalenas), chocolates, snacks salados (queso, maíz, galletitas saladas, papas fritas), galletitas dulces, bebidas dulces (jugos/gaseosas/bebidadeportistas). En confitería, 8/13 tenían exceso de GT, 10/13 de GS, 4/13 de GTr, 11/13, de AL, ninguno exceso de sodio, 11/13 tenían EA. Entre chocolates, todos tenían exceso de GT y GS, 2/10 en GTr, ninguno sodio, 9/10 altos en AL, 2/10 contenían EA. Snacks:, 14/17 eran altos en GT, 7/17 en GS, 1/17 en GTr, 15/17 en sodio, 4/17 en AL, 6/17 contenían EA. Galletitas dulces tenían exceso de GT 14/20, de GS 17/20, 6/20 de GTr, de sodio 1/20, todos con AL,17/20 contenían EA. En BDH

\section{ABSTRACT}

Introduction: Obesity in children is also associated with intake of foods high in fat, sugar and sodium, so it is important to know their levels in sweets and sweet drinks. Objectives: To evaluate critical nutrient content according to the PAHO Nutrient Profile in sweet treats, salty snacks and packaged non-alcoholic sweet drinks, according to nutritional labeling information. Materials and Methods: This was a cross-sectional, descriptive, exploratory study. Content per serving of total fat (TF), saturated fats (SF) and trans-fatty acids (TrF), sodium, free sugars (FS) and artificial sweeteners (AS) in sweet, salty, and nonalcoholic sweet drinks (NASD) was evaluated according to nutritional labeling. Excessive levels were considered according to the "PAHO Nutrient Profile". Samples were obtained for convenience at two outlet stores. Packaged desserts and treats with poorly visible information were excluded. Results: We evaluated 104 foods, which were grouped in: baked foods (alfajor, muffins), chocolates, salty snacks (cheese, corn, crackers, french fries), sweet cookies, and sweet drinks (juices / soft drinks / sports drinks). In baked foods, $8 / 13$ had excess of TF, 10/13 of SF, 4/13 of $\operatorname{TrF}, 11 / 13$ of FS, no excess of sodium, 11/13 had AS. Among chocolates, all had excess of TF and SF, 2/10 in TrF, none of sodium, 9/10 were high in FS, 2/10 contained AS. Snacks: $14 / 17$ were high in TF, $7 / 17$ in SF, $1 / 17$ in $\operatorname{TrF}, 15 / 17$ in sodium, 4/17 in FS, 6/17 contained AS. Sweet cookies had excess TF (14/20), SF (17/20), 6/20 had excess of TrF, sodium (1/20), all had excess FS, 17/20 contained AS. In NASD $(n=44)$, all had excess sodium, 36/44 had excess FS,

\footnotetext{
${ }^{1}$ Universidad Nacional de Asunción, Facultad de Ciencias Químicas. San Lorenzo, Paraguay.

Correspondencia:Susana F. Sánchez Bernal Correo: susana.sanchez.ber@gmail.com

Conflicto de interés: Los autores declaran no poseer conflicto de interés

Recibido: 08/02/2019Aceptado: 20/02/2020

DOI: https://doi.org/10.31698/ped.47012020006
}

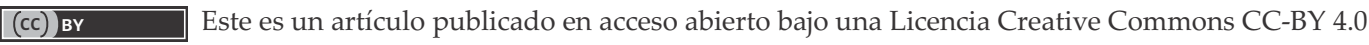


$(n=44)$, todas tenían exceso de sodio, 36/44 exceso de AL, 21/44 tenían EA. Conclusión: Alimentos de confitería son altos en grasas, azúcares libres y edulcorantes.. Los chocolates son altos en grasas y azucares. Los snacks son altos en sodio y grasas. Las galletitas tienen exceso de azúcares y grasas saturadas, 2/3 contienen edulcorantes artificiales. Las bebidas dulces son altas en sodio y azucares, la mitad tiene edulcorantes artificiales.

Palabras claves: Golosinas, bebidas, grasas, azúcares.
21/44 had AS. Conclusion: Baked foods are high in fat, free sugars and sweeteners. Chocolates are high in fats and sugars. Snacks are high in sodium and fat. Cookies have excess sugar and saturated fat, 2/3 contained artificial sweeteners. Sweet drinks are high in sodium and sugars, half have artificial sweeteners.

Keywords: Candies, drinks, fats, sugars.

\section{INTRODUCCIÓN}

El aumento progresivo de la obesidad global es una realidad a nivel mundial y tiene efectos sobre la salud y la economía mundial ${ }^{(1)}$. Organizaciones de salud alrededor del mundo coinciden en que los principales factores que promueven el exceso de peso y las enfermedades crónicas no trasmisibles relacionadas a la nutrición son la ingesta alta de alimentos ricos en azúcares, grasas y sal principalmente asociadas a alimentos ultraprocesas, o a la actividad física insuficiente ${ }^{(2)}$.

La población infantil no es ajena a esta realidad e inclusive es la más vulnerable a la frecuente exposición a publicidad o marketing de alimentos dirigida puesto que a estas edades, aún no tienen criterio propio para decidir sobre lo que es mejor para su salud e influencia fuertemente con campañas de marketing bien preparadas para atrapar a este sector de la población vulnerable ${ }^{(3,4)}$.

Entre los alimentos envasados frecuentemente promocionados para los niños se encuentran las golosinas y las bebidas dulces no alcohólicas. Las golosinas definidas como "alimentos industriales, nutricionalmente desbalanceados y con un alto contenido de hidratos de carbono, grasas o sal, densos en energía y pobres en nutrientes o altos en calorías y de baja densidad de nutrientes, o que contribuyen a la ingesta energética pero no en nutrientes" y pueden tener tanto sabor dulce como salado ${ }^{(5)}$.

La palatabilidad de estos productos predispone a su consumo en exceso especialmente en la población infantil $^{(6)}$, y por su alto tenor calórico aumenta el riesgo de sobrepeso, obesidad a corto y mediano plazo y esto a su vez, aumenta el riesgo de obesidad en la adultez $z^{(7)}$

Es altamente probable que la población general desconozca lo que realmente consumen los niños al ingerir golosinas o bebidas dulces y esta adquisición temprana de alimentación poco deseable con estos productos tiende a mantenerse en el tiempo ${ }^{(8)}$.

En esta línea, varios países han optado por la implementación de etiquetados frontales en productos envasados para advertir sobre el alto contenido de nutrientes como las grasas saturadas, grasas trans, azúcares libres y sodio entre otros, que son considerados como críticos para la nutrición y la salud $^{(9)}$.

El desafío en este sentido es contar con una referencia que establezca nivel de adecuación o de exceso de estos nutrientes críticos, ante lo cual la Organización Mundial de la Salud (OMS) elaboró un modelo de "Perfil de Nutrientes"(10) en el que se proponen valores límites para categorizar alimentos con excesivo contenido de dichos nutrientes.

En base a lo expuesto, el presente trabajo fue diseñado para evaluar la adecuación del contenido de sodio, grasas totales, grasas saturadas, grasas trans, azúcares y edulcorantes declarado en el etiquetado nutricional de envases de golosinas, snack salados y en bebidas dulces no alcohólicas, conforme a los valores propuestos en el Perfil de 
nutrientes de la Organización Panamericana de la Salud (OPS) $)^{(10)}$.

\section{MATERIALES Y MÉTODOS}

Estudio observacional descriptivo transversal, de carácter exploratorio en el que fueron incluidos productos alimenticios considerados "golosinas" (dulces y saladas) así como también bebidas dulces no alcohólicas.

La muestra estuvo constituida por todos los productos alimenticios envasados que que estaban disponibles para venta a todo público en una cadena de supermercados en Asunción entre los meses de setiembre a diciembre del año 2018 excluyéndose a los caramelos por la poca visibilidad del etiquetado y también a los postres envasados. El muestreo fue no probabilístico por conveniencia.

Para fines de este estudio los productos fueron organizados en grupos de acuerdo a la similitud de sus características de la siguiente manera:

- Productos alimenticios de confitería ${ }^{(11)}$ en el que se incluyeron: alfajores, confites rellenos, barrita de cereal, magdalena, barra rellena, mini torta, turrón de maní.

- Productos alimenticios de chocolate y a base de chocolate $^{(12)}$ fueron incluidos los chocolates en tableta, chocolates en barra, bombones, almendras cubiertas con chocolate y chocolate confitado.

- Galletas (o galletitas) dulces ${ }^{(13)}$ : en este grupo fueron incluidas galletitas rellenas y sin relleno, galletitas chips, galletas de avena, galletitas minichips.

- Snacks salados ${ }^{(14)}$ que abarcaron a: bocaditos de maíz, bocaditos a base de harina de papa, nuggets de pollo, formados de pechuga de pollo, producto de copetín, galletitas saladas, palos de harina de maíz, tortilla de maíz, mandiocas fritas, papas fritas.

- Bebidas dulces no alcohólicas ${ }^{(15)}$ : incluyó a bebidas gasificadas o carbonatadas (regulares, diet, free o zero), bebidas no gasificadas tales como jugos o néctares, bebidas a base de soja, y bebidas con adición de electrolitos.
Se realizó la evaluación de calorías y de nutrientes tales como grasas totales, grasas saturadas, grasas trans, azúcar libre agregada y sodio declarados en el etiquetado nutricional por 100 gramos de porción comestible y por porción de alimento recomendado en el envase. En el caso de los líquidos la composición correspondió al contenido por cada 100 mililitros. Estos valores luego fueron cotejados con los valores límites considerados "excesivos" acorde a lo establecido en el Modelo de Perfiles de Alimentos de la Organización Panamericana de la Salud ${ }^{(10)}$, según se presentan en el siguiente Figura1.

Figura 1. Criterios del modelo perfil de nutrientes de la OPS, utilizado para la evaluación de la adecuación de calorías y nutrientes de los etiquetados.

$\begin{aligned} & \text { Panel C. Criterios del modelo de perfil de nutrientes de la OPS para } \\
& \text { indicar los productos procesados y ultraprocesados que contienen una } \\
& \text { cantidad excesiva de sodio, azúcares libres, otros edulcorantes, grasas } \\
& \text { saturadas, total de grasas y grasas trans }\end{aligned}$
\begin{tabular}{c|l|l|l|l|l|} 
Sodio & $\begin{array}{l}\text { Azúcares } \\
\text { libres }\end{array}$ & $\begin{array}{c}\text { Otros } \\
\text { edulcorantes }\end{array}$ & $\begin{array}{c}\text { Total de } \\
\text { grasas }\end{array}$ & $\begin{array}{c}\text { Grasas } \\
\text { saturadas }\end{array}$ & $\begin{array}{c}\text { Grasas } \\
\text { trans }\end{array}$ \\
\hline $\begin{array}{l}\geq 1 \mathrm{mg} \text { de } \\
\text { sodio por } 1 \\
\text { kcal }\end{array}$ & $\begin{array}{l}\geq 10 \% \text { del } \\
\text { total de } \\
\text { energía } \\
\text { proveniente } \\
\text { de azúcares } \\
\text { libres }\end{array}$ & $\begin{array}{l}\text { Cualquier } \\
\text { cantidad de } \\
\text { otros } \\
\text { edulcorante } \\
\mathrm{s}\end{array}$ & $\begin{array}{l}\geq 30 \% \text { del } \\
\text { total de } \\
\text { energía } \\
\text { proveniente } \\
\text { del total de } \\
\text { grasas }\end{array}$ & $\begin{array}{l}\geq 10 \% \text { del } \\
\text { total de } \\
\text { energía } \\
\text { proveniente } \\
\text { de grasas } \\
\text { saturadas }\end{array}$ & $\begin{array}{l}\geq 1 \% \text { del } \\
\text { total de } \\
\text { energía } \\
\text { proveniente } \\
\text { de grasas } \\
\text { trans }\end{array}$ \\
\hline
\end{tabular}

Fuente: Modelo de perfil de nutrientes de la Organización Panamericana de la Salud.

Los datos fueron digitalizados en una planilla electrónica de Excel Microsoft Office ${ }^{\circledR} 2010$ y analizados en el software estadístico SPSS ${ }^{\circledR}$ V. 20.

Los datos cuantitativos se presentan en medianas, rango intercuartil y valores límites (mínimo y máximo), o en promedios y desviación estándar según distribución homogénea o no de los datos, determinados por la prueba de KolmogórovSmirnov. En caso de variables categóricas éstas se exponen en frecuencias y porcentajes.

El estudio contó con aprobación del Comité de Ética de la Facultad de Ciencias Químicas de la Universidad Nacional de Asunción.

\section{RESULTADOS}

En el estudio fueron incluidos 104 productos alimenticios considerados como golosinas tanto dulces como saladas, así como también bebidas dulces adquiridas de góndolas de venta en dos 
locales de una cadena de supermercados y tal como se presenta en la Tabla 1.

Tabla 1. Distribución de alimentos envasados dulces y salados por grupos estudiados.

\begin{tabular}{|c|c|c|}
\hline \multicolumn{3}{|c|}{ Alimentos envasados dulces } \\
\hline Agrupación & $\mathbf{n}$ & $\%$ \\
\hline Bebidas dulces no alcohólicas & 44 & 42,3 \\
\hline Confitería & 13 & 12,5 \\
\hline Chocolates & 10 & 9,6 \\
\hline Galletitas dulces & 20 & 19,2 \\
\hline Sub Total & 87 & 83,6 \\
\hline \multicolumn{3}{|c|}{ Alimentos envasados salados } \\
\hline Snacks salados & 17 & 16,3 \\
\hline Sub Total & 17 & 16,3 \\
\hline Total & 104 & 100 \\
\hline
\end{tabular}

En cuanto al cumplimiento del perfil de alimentos de la OPS se analizaron como críticos a las grasas totales, grasas saturadas, grasas trans, azúcares y sodio, y la presencia de edulcorantes artificiales en cada agrupación de alimentos.

En el grupo de alimentos tipo confitería fueron incluidos 13 productos alimenticios. En la Tabla 2 se presenta los valores en mediana de macro y micronutrientes estudiados. Se menciona que en cuanto al contenido de kilocalorías (kcal), la mediana por porción fue de $202 \mathrm{kcal}$ en tanto que el promedio de proteínas fue de 2,5 g y 3,4 de grasas totales.

Tabla 2. Contenido de macronutrientes, azúcares y sodio en productos alimenticios de confitería.

\begin{tabular}{|c|c|c|c|c|c|c|c|c|c|c|c|c|c|c|}
\hline \multirow[b]{2}{*}{ Contenido } & \multicolumn{2}{|c|}{ Kilocalorías } & \multicolumn{2}{|c|}{$\begin{array}{c}\text { Hidratos de } \\
\text { Carbono }\end{array}$} & \multicolumn{2}{|c|}{ Azúcares } & \multicolumn{2}{|c|}{ Proteínas } & \multicolumn{2}{|c|}{ Grasas totales } & \multicolumn{2}{|c|}{ Grasas Saturadas } & \multicolumn{2}{|c|}{ Sodio } \\
\hline & $\begin{array}{l}\text { Por } \\
\text { 100g }\end{array}$ & $\begin{array}{c}\text { Por } \\
\text { porción }\end{array}$ & $\begin{array}{c}\text { Por } \\
100 \mathrm{~g}\end{array}$ & $\begin{array}{c}\text { Por } \\
\text { porción }\end{array}$ & $\begin{array}{l}\text { Por } \\
100 \mathrm{~g}\end{array}$ & $\begin{array}{c}\text { Por } \\
\text { porción }\end{array}$ & $\begin{array}{c}\text { Por } \\
100 \mathrm{~g}\end{array}$ & $\begin{array}{c}\text { Por } \\
\text { porción }\end{array}$ & $\begin{array}{c}\text { Por } \\
100 \mathrm{~g}\end{array}$ & $\begin{array}{c}\text { Por } \\
\text { porción }\end{array}$ & $\begin{array}{c}\text { Por } \\
100 \mathrm{~g}\end{array}$ & $\begin{array}{c}\text { Por } \\
\text { porción }\end{array}$ & $\begin{array}{c}\text { Por } \\
100 \mathrm{~g}\end{array}$ & $\begin{array}{c}\text { Por } \\
\text { porción }\end{array}$ \\
\hline Mediana & 433 & 202 & 65,0 & 23,0 & 40,0 & 14,0 & $7,2 \pm 1,5^{*}$ & $2,5 \pm 1,2^{*}$ & $17,6 \pm 7,6^{*}$ & $6,9 \pm 3,5^{*}$ & $8,1 \pm 4,2 *$ & $3,4 \pm 2,2 *$ & $129,2 \pm 56,2^{*}$ & $54,9 \pm 37,1^{*}$ \\
\hline \multicolumn{15}{|l|}{ Cuartiles } \\
\hline Q1 & 394 & 112,5 & 58,0 & 16,0 & 37,0 & 9,0 & & & & & & & & \\
\hline Q2 & 433 & 202 & 65,0 & 23,0 & 40,0 & 14,0 & & & & & & & & \\
\hline Q3 & 526 & 234 & 69,5 & 33,0 & 48,5 & 19,1 & & & & & & & & \\
\hline Mínimo & 353 & 98 & 32,5 & 13,0 & 0,0 & 0,0 & 5,0 & 1,0 & 8,0 & 2,0 & 1,3 & 0,3 & 49,0 & 12,0 \\
\hline Máximo & 1050 & 420 & 166,8 & 42,0 & 615,4 & 27,0 & 9,5 & 4,9 & 30,0 & 14,0 & 14,0 & 8,1 & 227,0 & 148,0 \\
\hline
\end{tabular}

En la Tabla 3 se presenta la adecuación de los nutrientes críticos evaluados en los productos de confitería. Se destaca que todos fueron adecuados en cuanto a niveles de Sodio, 10 productos tenían exceso de grasas saturadas y 11 presentaron exceso de azúcares libres.

En el grupo de chocolates se incluyó un total de 10 productos alimenticios. Para esta agrupación la mediana de kcal por porción fue de 142,3 kcal y tenían 2 g y 16,5 g de grasas saturadas (Tabla 4).
Tabla 3. Adecuación de Sodio, grasas, azúcares y de edulcorantes en productos alimenticios de tipo confitería según Perfil de Alimentos de la OPS (n=13).

\begin{tabular}{llcc}
\hline Contenido & Categoría & N & (\%) \\
\hline Grasas totales & Adecuado & 5 & 38,5 \\
& Excesivo & 8 & 61,5 \\
Grasas saturadas & Adecuado & 3 & 23,1 \\
& Excesivo & 10 & 76,9 \\
\hline \multirow{2}{*}{ Grasas trans } & Adecuado & 9 & 69,2 \\
& Excesivo & 4 & 30,8 \\
Sodio & Adecuado & 13 & 100,0 \\
& Excesivo & 0 & - \\
Azúcares libres & Adecuado & 2 & 15,4 \\
\multirow{2}{*}{ Edulcorantes } & Excesivo & 11 & 84,6 \\
& Tiene & 10 & 76,9 \\
TOTAL & No tiene & 3 & 23,1 \\
\hline
\end{tabular}

Tabla 4. Contenido de macronutrientes, azúcares y sodio en productos alimenticios tipo chocolates por porción y por 100 gramos $(n=10)$.

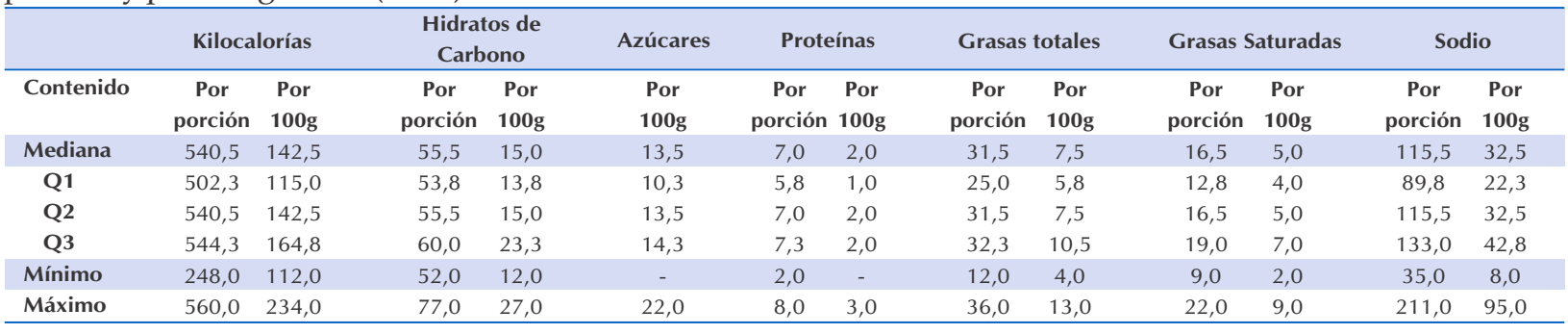


El nivel de las grasas totales fue excesivo en los 10 productos de chocolate, también el nivel de grasas saturadas y el nivel de azúcares libres fue excesivo en 9 de los productos según el Perfil de Alimentos de la OPS tal como se presenta en la Tabla 5.

En el grupo de galletitas dulces con total de 20 productos se observó que la mediana de kilocalorías por porción fue de 140,5 Kcal y de 9,5 g para los azucares libres mientras que la de proteínas fue de $2 \mathrm{~g}$ (Tabla 6).
Tabla 5. Adecuación de Sodio, grasas, azúcares y de edulcorantes en productos alimenticios del grupo de chocolates según Perfil de Alimentos de la OPS ( $\mathrm{n}=10)$

\begin{tabular}{llcc}
\hline Contenido & Categoría & Frecuencia & Porcentaje \\
\hline Grasas totales & Adecuado & 0 & 0 \\
& Excesivo & 10 & 100 \\
Grasas saturadas & Adecuado & 0 & 0 \\
& Excesivo & 10 & 100 \\
Grasas trans & Adecuado & 8 & 80 \\
& Excesivo & 2 & 20 \\
Sodio & Adecuado & 10 & 100 \\
& Excesivo & 0 & 0 \\
Azúcares libres & Adecuado & 1 & 10 \\
\multirow{2}{*}{ Edulcorantes } & Excesivo & 9 & 90 \\
& Tiene & 2 & 20 \\
TOTAL & No tiene & 8 & 80 \\
\hline & & 10 & \\
\hline
\end{tabular}

Tabla 6. Contenido de macronutrientes, azúcares y sodio en galletitas dulces por porción y por 100 gramos $(n=20)$.

\begin{tabular}{|c|c|c|c|c|c|c|c|c|c|c|c|c|c|}
\hline \multirow[b]{2}{*}{ Contenido } & \multicolumn{2}{|c|}{ Kilocalorías } & \multicolumn{2}{|c|}{$\begin{array}{c}\text { Hidratos de } \\
\text { Carbono } \\
\end{array}$} & \multirow{2}{*}{$\begin{array}{c}\text { Azúcares } \\
\text { Por } \\
100 \mathrm{~g}\end{array}$} & \multicolumn{2}{|c|}{ Proteínas } & \multicolumn{2}{|c|}{ Grasas totales } & \multicolumn{2}{|c|}{ Grasas Saturadas } & \multicolumn{2}{|c|}{ Sodio } \\
\hline & $\begin{array}{c}\text { Por } \\
\text { porción }\end{array}$ & $\begin{array}{c}\text { Por } \\
100 \mathrm{~g}\end{array}$ & $\begin{array}{c}\text { Por } \\
\text { porción }\end{array}$ & $\begin{array}{l}\text { Por } \\
100 \mathrm{~g}\end{array}$ & & $\begin{array}{c}\text { Por } \\
\text { porción }\end{array}$ & $\begin{array}{c}\text { Por } \\
100 g\end{array}$ & $\begin{array}{c}\text { Por } \\
\text { porción }\end{array}$ & $\begin{array}{c}\text { Por } \\
100 g\end{array}$ & $\begin{array}{c}\text { Por } \\
\text { porción }\end{array}$ & $\begin{array}{l}\text { Por } \\
100 \mathrm{~g}\end{array}$ & $\begin{array}{c}\text { Por } \\
\text { porción }\end{array}$ & $\begin{array}{c}\text { Por } \\
\text { 100g }\end{array}$ \\
\hline Mediana & 465,0 & 140,5 & 69,0 & 21,0 & 9,5 & 7,0 & 2,0 & 18,0 & 5,0 & 7,5 & 2,0 & 274,0 & 82,5 \\
\hline Q1 & 440,8 & 131,3 & 66,3 & 20,0 & 7,0 & 5,0 & 1,0 & 15,0 & 4,0 & 5,3 & 1,0 & 221,0 & 67,5 \\
\hline Q2 & 465,0 & 140,5 & 69,0 & 21,0 & 9,5 & 7,0 & 2,0 & 18,0 & 5,0 & 7,5 & 2,0 & 274,0 & 82,5 \\
\hline Q3 & 481,3 & 159,0 & 70,8 & 24,5 & 11,8 & 7,8 & 2,0 & 19,0 & 5,8 & 10,0 & 3,0 & 318,3 & 110,3 \\
\hline Máximo & 539,0 & 251,0 & 89,0 & 35,0 & 17,0 & 9,0 & 3,0 & 24,0 & 11,0 & 13,0 & 6,0 & 444,0 & 160,0 \\
\hline
\end{tabular}

Con respecto a la adecuación de los niveles de nutrientes críticos estudiados el nivel de las grasas totales fue excesivo en las 20 galletitas dulces, en 17/20 productos el nivel de grasas saturadas fue excesivo, el nivel de azúcares libres fue excesivo en todas las galletitas y 17/20 contenían edulcorantes artificiales.

En el grupo de snacks salados fueron incluidos 16 productos cuya mediana de kilocalorías por porción fue de 123,5 y la de grasas totales por porción fue de $5,5 \mathrm{~g}$ de las guales $3 \mathrm{~g}$ eran saturadas (Tabla 8) y la del Sodio fue de $161 \mathrm{mg}$ con un máximo de hasta 718 $\mathrm{mg} /$ porción.
Tabla 7. Adecuación de Sodio, grasas, azúcares y de edulcorantes en galletitas dulces según Perfil de Alimentos de la OPS ( $\mathrm{n}=20)$.

\begin{tabular}{llcc}
\hline Contenido & Categoría & Frecuencia & Porcentaje \\
\hline Grasas totales & Adecuado & 6 & 30 \\
& Excesivo & 14 & 70 \\
Grasas saturadas & Adecuado & 3 & 15 \\
& Excesivo & 17 & 85 \\
Grasas trans & Adecuado & 14 & 70 \\
& Excesivo & 6 & 30 \\
Sodio & Adecuado & 19 & 95 \\
& Excesivo & 1 & 5 \\
Azúcares libres & Adecuado & 0 & 0 \\
& Excesivo & 20 & 100 \\
Edulcorantes & Tiene & 17 & 85 \\
& No tiene & 3 & 15 \\
TOTAL & & 20 & 100 \\
\hline
\end{tabular}

Tabla 8. Contenido de macronutrientes, azúcares y sodio en productos alimenticios tipo snack salado por porción y por 100 gramos $(\mathrm{n}=16)$.

\begin{tabular}{|c|c|c|c|c|c|c|c|c|c|c|c|c|c|c|c|c|}
\hline \multirow[b]{2}{*}{ Contenido } & \multicolumn{2}{|c|}{$\begin{array}{c}\text { Calorías totales } \\
\text { (kcal) }\end{array}$} & \multicolumn{2}{|c|}{$\begin{array}{l}\text { Hidratos de } \\
\text { Carbono (g) }\end{array}$} & \multicolumn{2}{|c|}{$\begin{array}{l}\text { Azúcares } \\
\text { (g) }\end{array}$} & \multicolumn{2}{|c|}{$\begin{array}{l}\text { Proteínas } \\
\text { (g) }\end{array}$} & \multicolumn{2}{|c|}{$\begin{array}{c}\text { Grasas totales } \\
\text { (g) }\end{array}$} & \multicolumn{2}{|c|}{$\begin{array}{c}\text { Grasas Saturadas } \\
\text { (g) }\end{array}$} & \multicolumn{2}{|c|}{$\begin{array}{c}\text { Grasas trans } \\
\text { (g) }\end{array}$} & \multicolumn{2}{|c|}{$\begin{array}{c}\text { Sodio } \\
\text { (mg) }\end{array}$} \\
\hline & $\begin{array}{l}\text { Por } \\
\text { 100g }\end{array}$ & $\begin{array}{c}\text { Por } \\
\text { porción }\end{array}$ & $\begin{array}{l}\text { Por } \\
100 \mathrm{~g}\end{array}$ & $\begin{array}{l}\text { Por } \\
\text { porción }\end{array}$ & $\begin{array}{l}\text { Por } \\
100 \mathrm{~g}\end{array}$ & $\begin{array}{l}\text { Por } \\
\text { oorción }\end{array}$ & $\begin{array}{l}\text { Por } \\
100 g\end{array}$ & $\begin{array}{c}\text { Por } \\
\text { porción }\end{array}$ & $\begin{array}{l}\text { Por } \\
100 g\end{array}$ & $\begin{array}{c}\text { Por } \\
\text { porción }\end{array}$ & $\begin{array}{l}\text { Por } \\
100 g\end{array}$ & $\begin{array}{c}\text { Por } \\
\text { porción }\end{array}$ & $\begin{array}{l}\text { Por } \\
100 \mathrm{~g}\end{array}$ & $\begin{array}{c}\text { Por } \\
\text { porción }\end{array}$ & $\begin{array}{l}\text { Por } \\
100 g\end{array}$ & $\begin{array}{c}\text { Por } \\
\text { porción }\end{array}$ \\
\hline Mediana & 473,0 & 119,0 & 64,0 & 17,0 & 0,0 & 0,5 & 6,4 & 1,7 & 20,0 & 5,5 & 3,2 & 0,8 & 0,0 & 0,0 & 600,0 & 156,0 \\
\hline Q1 & 439,0 & 115,0 & 53,5 & 15,0 & 0,0 & 0,0 & 5,6 & 1,4 & 12,9 & 4,8 & 2,3 & 0,6 & 0,0 & 0,0 & 500,0 & 136,0 \\
\hline Q2 & 473,0 & 119,0 & 64,0 & 17,0 & 0,0 & 0,5 & 6,4 & 1,7 & 20,0 & 5,5 & 3,2 & 0,8 & 0,0 & 0,0 & 600,0 & 156,0 \\
\hline Mínimo & 89,2 & 93,0 & 5,9 & 7,7 & 0,0 & 0,0 & 4,0 & 1,0 & 0,8 & 1,1 & 0,0 & 0,0 & 0,0 & 0,0 & 369,2 & 120,0 \\
\hline Máximo & 560,0 & 283,0 & 88,0 & 22,0 & 300,0 & 10,7 & 18,5 & 24,0 & 33,0 & 11,0 & 12,0 & 4,0 & 2,4 & 0,6 & 1235,0 & 718,0 \\
\hline
\end{tabular}


Al evaluar la adecuación de nutrientes críticos, se observó que el nivel de las grasas totales fue excesivo en 14/16 de los productos, el nivel de grasas saturadas fue excesivo en 7/16 y 14/16 productos con nivel excesivo de Sodio tal como se observa en la Tabla 9.

En el grupo de bebidas dulces no alcohólicas fueron evaluados 44 productos. El promedio de calorías para este grupo porción fue de $58,5 \pm 33 \mathrm{kcal}$, el promedio de azúcares libres fue de de $12,9 \mathrm{~g}$ por porción. Por otra parte, la mediana del contenido de Sodio fue de $23 \mathrm{mg}$ por porción tal como se observa en la Tabla 10.
Tabla 9. Adecuación de Sodio, grasas, azúcares y de edulcorantes en productos alimenticios tipo snack salado según Perfil de Alimentos de la OPS (n=16).

\begin{tabular}{llcc}
\hline Contenido & Categoría & Frecuencia & Porcentaje \\
\hline Grasas totales & Adecuado & 3 & 17,6 \\
& Excesivo & 14 & 82,4 \\
Grasas saturadas & Adecuado & 10 & 58,8 \\
& Excesivo & 7 & 41,2 \\
Grasas trans & Adecuado & 16 & 94,1 \\
& Excesivo & 1 & 5,9 \\
Sodio & Adecuado & 2 & 11,8 \\
& Excesivo & 15 & 88,2 \\
Azúcares libres & Adecuado & 13 & 76,5 \\
& Excesivo & 4 & 23,5 \\
Edulcorantes & Tiene & 6 & 35,3 \\
& No tiene & 11 & 64,7 \\
\hline TOTAL & & 17 & \\
\hline
\end{tabular}

Tabla 10. Contenido de macronutrientes, azúcares y sodio en productos alimenticios en bebidas azucaradas por porción y por 100 gramos $(\mathrm{n}=44)$.

\begin{tabular}{|c|c|c|c|c|c|c|c|c|c|}
\hline \multirow[b]{2}{*}{ Contenido } & \multicolumn{2}{|c|}{$\begin{array}{c}\text { Calorías } \\
\text { (kcal) }\end{array}$} & \multicolumn{2}{|c|}{$\begin{array}{l}\text { Hidratos de } \\
\text { Carbono (g) }\end{array}$} & \multicolumn{2}{|c|}{$\begin{array}{l}\text { Azúcares libres } \\
\text { (g) }\end{array}$} & \multicolumn{2}{|r|}{$\begin{array}{c}\text { Sodio } \\
\text { (mg) }\end{array}$} & \multirow[b]{2}{*}{$\begin{array}{l}\text { Por } \\
\text { porción }\end{array}$} \\
\hline & $\begin{array}{l}\text { Por } \\
100 g\end{array}$ & $\begin{array}{c}\text { Por } \\
\text { porción }\end{array}$ & $\begin{array}{l}\text { Por } \\
100 g\end{array}$ & $\begin{array}{c}\text { Por } \\
\text { porción }\end{array}$ & $\begin{array}{l}\text { Por } \\
100 \mathrm{~g}\end{array}$ & $\begin{array}{c}\text { Por } \\
\text { porción }\end{array}$ & $\begin{array}{l}\text { Por } \\
100\end{array}$ & & \\
\hline Promedio & 29,3 & 58,5 & 7,1 & 14,2 & 6,5 & 12,9 & 11,5 & & $23^{*}$ \\
\hline Desvío Estándar & 16,4 & 33,0 & 4,1 & 8,2 & 4,2 & 8,3 & $\begin{array}{l}\text { Q1 } \\
\text { Q2 } \\
\text { Q3 }\end{array}$ & $\begin{array}{l}6,1 \\
11,5 \\
25(* *)\end{array}$ & $\begin{array}{ll}\text { Q1 } & 12,3 \\
\text { Q2 } & 23 \\
\text { Q3 } & 49,3(* *)\end{array}$ \\
\hline Mínimo & 0,0 & 0,0 & 0,0 & 0,0 & 0,0 & 0,0 & 2,2 & & 4,3 \\
\hline Máximo & 54,0 & 108,0 & 13,5 & 27,0 & 13,5 & 27,0 & 63,0 & & 126,0 \\
\hline
\end{tabular}

Con respecto al nivel de sodio, fue excesivo en las 44 bebidas estudiadas, como era de esperar no contenían grasa y el nivel de azúcares libres fue excesivo en $36 / 44$ productos y $21 / 44$ declaraban contener edulcorantes artificiales (Tabla 11).

Tabla 11. Adecuación de Sodio, grasas, azúcares y de edulcorantes en bebidas azucaradas según Perfil de Alimentos de la OPS ( $\mathrm{n}=45)$.

\begin{tabular}{llcc}
\hline Contenido & Categoría & Frecuencia & Porcentaje \\
\hline Sodio & Adecuado & 0 & 0 \\
& Excesivo & 44 & 100 \\
Azúcares libres & Adecuado & 8 & 97,8 \\
& Excesivo & 36 & 2,2 \\
Edulcorantes & Tiene & 21 & 48,9 \\
& No tiene & 23 & 51,1 \\
TOTAL & & 44 & \\
\hline
\end{tabular}

Todos los productos incluidos en el estudio contaban con datos del número de Registro Sanitario de Producto Alimenticio (RSPA) así como también cumplían todos con la normativa MERCOSUR referente al rotulado nutricional de productos envasados.

\section{DISCUSIÓN}

Hemos encontrado que la mayoría de las golosinas dulces y snacks salados tienen excesivo contenido de grasas totales y saturadas, y que todas las bebidas dulces, incluso las consideradas como "jugos", tienen sodio en exceso y una proporción importante de ellas, exceso de azúcares libres según valores del Perfil de nutrientes de alimentos de la OPS ${ }^{(10)}$.

Nuestro estudio revela que varios productos alimenticios que son de venta libre y algunos a bajo costo, cuyo marketing publicitario en su mayoría está dirigido principalmente hacia los niños y niñas tal como lo demuestra el estudio realizado por Castillo-Lancellotti y cols. ${ }^{(16)}$ en el cual observaron un predominio de publicidad dirigida a niños asociada a alimentos y bebidas no saludables.

Precisamente la estrategia publicitaria utiliza principalmente la técnica de marketing de la diversión asociada al consumo de alimentos y bebidas según lo evaluado por Goméz y $\operatorname{cols}^{(17)}$ demostrando que además el atributo principal al que apelan es la palatabilidad de los productos alimenticios publicitados.

El problema se presenta cuando a la par del aumento de productos ultra procesados como lo son las 
golosinas y snacks, los cuales colaboran en proporción importante a una dieta no saludable, aumenta también la prevalencia de sobrepeso y obesidad infantil ${ }^{(8)}$.

Si bien nuestro trabajo no evaluó el consumo de los productos alimenticios estudiados o el volumen de bebidas dulces ingeridas. el estudio realizado en nuestro país por Aguilar y $\operatorname{cols}^{(18)}$, demostró que niños y adolescentes con exceso de peso, beben mayor volumen de jugos artificiales y bebidas carbonatadas (gaseosas) que sus pares con índice de masa corporal normal. Este volumen mayor implicaría mayor cantidad de azúcares y de sodio acorde a lo hallado en nuestro estudio, lo cual es similar a estudios realizados en la región ${ }^{(19)}$ y a lo estudiado por Imamura y cols. ${ }^{(20)}$.

El exceso de sodio hallado en las bebidas dulces y en los snacks evaluados, pudieran a corto y mediano plazo potenciar el desarrollo de hipertensión $\operatorname{arterial}^{(21)}$, más aún cuando fueran consumidos ya desde la temprana infancia.

Algo que llama la atención es la adición de edulcorantes artificiales, en productos que no esperábamos que lo contuvieran tales como productos de confitería y las galletas o galletitas dulces presentaban niveles suficientes como para ser declarados en el etiquetado nutricional al igual a lo encontrado en las bebidas dulces no alcohólicas estudiadas y que no en todos los casos eran declaradas como light o diet. La sola adición de edulcorantes artificiales a los productos alimenticios, los hace no recomendables para la población infantil salvo casos especiales ${ }^{(22)}$.

Entre las limitaciones del presente estudio se mencionan fundamentalmente la imposibilidad de llegar a la totalidad de los productos disponibles ya que se dificultó el acceso a todos los puntos de ventas existentes. Sin embargo, previamente se realizó un sondeo para evaluar la oferta de productos esperados, no hallándose mayores diferencias en la misma. Además, la limitación en la visibilidad del etiquetado en productos tan pequeño como por ejemplo los caramelos, impidió su evaluación por lo cual se sugiere que a futuro sean revisadas las fichas técnicas de los mismos en la entidad competente que habilita su comercialización.

Por otra parte, también se menciona que sería importante realizar un análisis de composición química de estos productos a fin de corroborar si concuerda con lo declarado en el etiquetado nutricional.

Sin embargo, a pesar de las limitaciones consideramos que es sumamente importante evidenciar la mala calidad nutricional que tienen las golosinas, snack y bebidas dulces frecuentemente consumidos por nuestros niños con este tipo de estudios ya que el consumo elevado de azúcares y grasas se asocia con diversas patologías como sobrepeso, obesidad, alteraciones hepáticas, desórdenes del comportamiento, diabetes, hiperlipidemia, enfermedad cardiovascular, hígado graso, algunos tipos de cáncer y caries dental ${ }^{(23)}, \mathrm{y}$ éstos nutrientes críticos se encuentran en exceso en los productos alimentarios evaluados en el presente estudio.

Esto nos lleva a plantear la necesidad urgente de que los padres o cuidadores tomen conciencia sobre lo que realmente está ofreciendo a sus niños y adolescentes a través de normativas que permitan que el ciudadano no precise de conocimientos técnicos para valorar la pobre calidad nutricional de ciertos productos. La estrategia más promocionada es el uso de etiquetado frontal con mensajes de advertencia ${ }^{(24)}$ cuyo formato debe ser llamativo y fácil de comprender ${ }^{(25)}$ y que desde hace un tiempo está generando evidencia de impacto en la elección y consumo de alimentos con alto nivel de nutrientes de riesgo para la salud ${ }^{(26)}$.

\section{CONCLUSIONES}

Las golosinas dulces en todos los grupos de alimentos estudiados contienen exceso de grasas saturadas, azúcares libres y edulcorantes en su mayoría, principalmente las galletitas dulces y los productos de confitería. Los snacks salados también contienen grasas saturadas en cantidad excesiva, pero además tienen alta cantidad sodio. Todas las bebidas dulces no alcohólicas tienen sodio y azúcares libres en exceso, y poco más de la mitad contienen edulcorantes artificiales. 


\section{REFERENCIAS BIBLIOGRAFICAS}

1. International Bank for Reconstruction and Development. Obesity: Health and Economic Consequences of an Impending Global Challenge. Meera S, Popkin B, eds. Washington, DC: World Bank Group; 2020.233 p.

2. Pan-American Health Organization (PAHO). Ultraprocessed food and drink products in Latin America: trends, impact on obesity, policy implications [Internet]. Washington, DC: PAHO; 2015. [Consultado 18 Dic 2018] Disponible en: http://iris.paho.org/xmlui/bitstream/hand le/123456789/7699/9789275118641_eng.pdf

3. Giménez A, Saldamando L de, Curutchet MR, Ares G. Package design and nutritional profile of foods targeted at children in supermarkets in Montevideo, Uruguay. Cad Saude Publica. 2017;33(5):1-11.

4. Bernhardt AM, Wilking C, Adachi-Mejia AM, Bergamini E, Marijnissen J, Sargent JD. How Television Fast Food Marketing Aimed at Children Compares with Adult Advertisements. PLoS One. 2013; 8(8):1-6.

5. Jackson P, Romo MM, Castilio AM, Castillo-Durán C. Las golosinas en la alimentación infantil. Análisis antropológico nutricional. Rev Med Chil. 2004; 132(10):1235-42.

6. Murray RD. Savoring Sweet: Sugars in Infant and Toddler Feeding Savoring Sweet : Sugars in Infant and Toddler Feeding. Ann Nutr Metab. 2017; 70(suppl3):38-46.

7. Simmonds M, Llewellyn A, Owen CG, Woolacott N. Predicting adult obesity from childhood obesity: a systematic review and meta-analysis. Obes Rev. 2016; 17(2):95-107.

8. Luque V, Escribano J, Closa-Monasterolo R, ZaragozaJordana M, Ferre N, Grote V, et al. Unhealthy dietary patterns established in infancy track to mid-childhood: the EU Childhood Obesity Project. J Nutr. 2018; 148(5):752-9.

9. Vanderlee L, White CM, Bordes I, Hobin EP, Hammond D. The efficacy of sugar labeling formats: Implications for labeling policy. Obesity. 2015;23(12):2406-2413.

10. Organización Panamericana de la Salud. Modelo de perfil de nutrientes de la Organización Panamericana de la Salud. Washington, DC: OPS, 2016.

11. A.A.P.P.A. Introducción a la Tecnología de Alimentos. Editorial Limusa, 2003.148 p.

12. FAO, OMS. Norma para el chocolate y los productos del chocolate: codex stan 87-1981. Adoptada en 1981. Revisión: 2003. Enmienda: 2016.
13. NMX-F-006-1983. Alimentos. Galletas. Food. Cookie: Normas Mexicanas. Dirección General de Normas; 2016. 14. Montoya R. Diccionario comentado del español actual en Colombia. 3ra. ed. Madrid: Editorial Visión Libros; 2006. 162 p.

15. FAO, WHO. Norma general del CODEX para zumos (jugos) y néctares de frutas: CODEX STAN 247. FAO; 2005.

16. Castillo-Lancellotti C, Pérez-Santiago O, Rivas-Castillo C, Fuentes-García R, Tur-Marí JA. Análisis de la publicidad de alimentos orientada a niños y adolescentes en canales chilenos de televisión abierta. Rev Esp Nutr Comunitaria. 2010; 16(2):90-97.

17. Gómez P, Zapata ME, Rovirosa A, Gotthelf S, Ferrante D. Técnicas de marketing en publicidades de alimentos y bebidas en canales infantiles de Argentina: diferencias según calidad nutricional. Rev Argent Salud Pública. 2017; 8(33): 22-27.

18. Aguilar G, Estigarribia G, Sanabria G, Sanabria M, Kawabata A, Munoz S, et al. Sobrepeso, obesidad e ingesta de líquidos en niños y adolescentes en Capital, Central y Caaguazu, 2016. Pediatr. (Asunción) [Internet]. 2018 [Consultado 20 Dic 2018]; 45(2): 147-154.

19. Teixeira ZA. Sodium content and food additives in major brands of Brazilian children's foods. Ciência \& Saúde Coletiva. 2018;23(12):4065-4075.

20. Imamura F, O'Connor L, Ye Z, Mursu J, Hayashino Y, Bhupathiraju SN, et al. Consumption of sugar sweetened beverages, artificially sweetened beverages, and fruit juice and incidence of type 2 diabetes: systematic review, metaanalysis, and estimation of population attributable fraction. BMJ. 2015;351:h3576. doi: 10.1136/bmj.h3576

21. Kim Y, Je Y. Prospective association of sugarsweetened and artificially sweetened beverage intake with risk of hypertension. Archives of Cardiovascular Diseases. 2016; (4):242-253.

22. Wakida-Kuzunoki G, Aguiñaga-Villaseñor RG, AvilésCobián R, Baeza-Bacab M, Cavagnari B, Castillo-Ruíz V, et al. Edulcorantes no calóricos en la edad pediátrica: análisis de la evidencia científica. Rev Mex Pediatr. 2017; 84(Supl. 1); S3-S23.

23. Cabezas-Zabala C, Hernández-Torres C, VargasZárate M. Azúcares adicionados a los alimentos: efectos en la salud y regulación mundial. Rev fac med. 2016; 64(2):319-329. 
24. Vanderlee L, White CM, Bordes I, Hobin EP, Hammond D. The efficacy of sugar labeling formats: Implications for labeling policy. Obesity. 2015;23:2406-2413.

25. Goodman S, Vanderlee L, Acton R, Mahamad S, Hammond D. The Impact of Front-of-Package Label Design on Consumer Understanding of Nutrient Amounts. Nutrients. 2018; 10(11):1624.
26. Ikonen I, Sotgiu F, Aydinli A, Verlegh P. Consumer effects of front-of-package nutrition labeling: an interdisciplinary meta-analysis. J. of the Acad. Mark. Sci. 2019; DOI: https://doi.org/10.1007/s11747-019-00663-9 